\title{
Evaluation of cytotoxicity, anti-herpes simplex virus type 1 (HSV-1) and antibacterial activities of Ficus vasta and phytoconstituents
}

\author{
*Khaled Rashed ${ }^{1}$, Lucy Ono ${ }^{2}$ \\ ${ }^{1}$ National Research Centre, Pharmacognosy Department, Dokki, Giza, Egypt \\ ${ }^{2}$ Yasuyoshi Hayashi Microbiology Laboratory, Department of Basic Pathology, UFPR, Curitiba, PR, Brazil
}

\begin{abstract}
This study deals with the evaluation of in vitro cytotoxicity of the methanol 80\% extract of Ficus vasta aerial parts in Vero cells, antiHSV-1 and antibacterial activities and the determination of the phytochemical content of the F. vasta methanol extract. The extract was tested for its cytotoxicity in Vero cells and for anti-HSV-1 and against bacterial strains as S. aureus, S. epidermidis, E. coli and P. aeruginosa and also phytochemical constituents were detected. The results showed that this extract presented a CC50 value of 389 $\mu \mathrm{g} / \mathrm{mL}$, an intermediate value taking into account other researchers reported for some Moraceae species. Regarding the other biological activities, antiviral effect was not demonstrated by a HSV-1 challenge dilution of 100x tissue culture infective dose 50\% $\left(\mathrm{TCID}_{50}\right)$ and also by the disk-diffusion method at the higher non-toxic concentration of the extract $(119 \mu \mathrm{g} / \mathrm{mL})$. Phytochemical analysis of the methanol extract proved the presence of carbohydrates, tannins, flavonoids, coumarins, alkaloids and triterpenes. Chromatographic separation of the methanol extract resulted in the isolation and identification of $\beta$-sitosterol, stigmasterol, lupeol, ursolic acid, naringenin, luteolin, quercetin, vitexin, quercetin $3-O-\beta$-glucoside and rutin. The results indicated that $F$. vasta aerial parts methanol extract is potential candidate for experiments of biological activity screening that are not anti-HSV-1 or antibacterial against the strains evaluated in this study, to be carried on in the future.
\end{abstract}

Key Words: Plant extract, biological activities, cytotoxicity, anti-HSV-1, antibacterial, phytoconstituents.

\section{INTRODUCTION}

Infectious diseases remain as an important worldwide health problem and the control of them is the subject of constant scientific endeavor. Additionally, the appearance of microbial strains resistant to chemotherapeutic agents is an emerging problem. The severe side effects and the emergence of drug-resistance mutants during long-term medication with these drugs have often limited their administration to patients (Bacon et al., 2003; Morfin and Thouvenot, 2003). Herbal remedies used in the traditional folk medicine provide an interesting and still largely unexplored source for the creation a development of potentially new drugs for chemo-therapy which might help to overcome the growing problem of resistance and also the toxicity of the currently available commercial drugs. The traditional medicinal methods, especially the use of medicinal plants, still play a vital role to cover the basic health needs in the developing countries (Awadh et al., 2001). Therefore, it is of great interest to carry out a screening of these plants in order to validate their use in folk medicine and to reveal the active principle by isolation and characterization of their constituents (Awadh et al., 2001) and thus, the evaluation of the cytotoxic potential of novel candidates to antimicrobial agents is an important area of research. In our searching for natural products as potential antimicrobial drugs, Ficus vasta is a very large tree from Moraceae family growing over $25 \mathrm{~m}$ tall. F. vasta is a tree of dry north and eastern Africa, Sudan, Ethiopia, Saudi Arabia and Tanzania (Dawit et al., 2003). In traditional medicine, F. vasta used in rheumatism, pains, intestinal worms (Kitessa

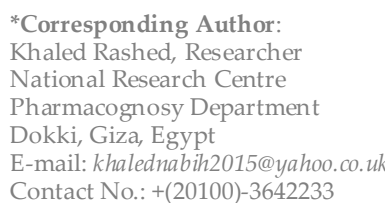

et al., 2007). It was already studied for the extract from the fruits of F. vasta antibacterial (Al-Fatimi et al., 2007), mainly against Gram-positive bacteria, and antihelmintic (Raju et al., 2011) activities. There are no previous reports about phytoconstitutents and the cytotoxicity against Vero cells, anti-HSV-1 and antibacterial activities from the aerial parts of $F$. vasta, which are now described in this research article.

\section{MATERIALS AND METHODS}

\section{Experimental}

UV/VIS: Shimadzu UV-visible recording spectrophotometer model-UV 240 (NRC, Egypt). ${ }^{1} \mathrm{H}-\mathrm{NMR}$ and ${ }^{13} \mathrm{C}-\mathrm{NMR}$ (Varian Unity Inova). MS (Finnigan MAT SSQ 7000, 70 ev). (Silica gel $(0.063-0.200 \mathrm{~mm}$ for column chromatography) and Sephadex LH-20 (Pharmacia Fine Chemicals). Thin layer chromatography (TLC) $\mathrm{F}_{254}$ plates. Solvent mixtures, BAW (n-butanol:acetic acid:water 4:1:5 upper phase, 15\% acetic acid: water: glacial acetic acid: 85:15). Paper Chromatography (PC) Whatman No.1 (Whatman Led. Maid Stone, Kent, England) sheets for qualitative detection of flavonoids and sugars.

\section{Plant identification and collection}

Ficus vasta aerial parts were collected from Al-Zohiriya garden, Giza, Egypt in May 2011. The plant was identified by Dr. Mohammed El-Gebaly, Department of Botany, National Research Centre (NRC) and by Mrs. Tereeza Labib Consultant of Plant Taxonomy at the Ministry of Agriculture and director of Orman botanical garden, Giza, Egypt. A voucher specimen was deposited in the herbarium of Al-Zohiriya garden, Giza, Egypt.

Preparation of $F$. vasta aerial parts methanol $80 \%$ extract Air dried powder of $F$. vasta aerial parts $(720 \mathrm{~g})$ was extracted with methanol : water $(80: 20)(\mathrm{v} / \mathrm{v})$ at room temperature several times until exhaustion by maceration 
Table 1: Compounds isolation result of methanol extract (36 g) of the F. vasta aerial parts. It was subjected to silica gel column chromatography and eluted with different amounts of hexane, dichloromethane, ethyl acetate and methanol.

\begin{tabular}{|c|c|c|c|}
\hline $\begin{array}{l}\text { Fraction } \\
\text { No. }\end{array}$ & $\underset{\text { (g) }}{\text { Amount }}$ & Mobile phase (ratio) & $\begin{array}{l}\text { Compound } \\
\text { No. }\end{array}$ \\
\hline \multirow[t]{2}{*}{1} & 1.64 & $\begin{array}{l}\text { n-hexane: dichloromethane } \\
(60: 40 \mathrm{v} / \mathrm{v})\end{array}$ & Comp. 1 \\
\hline & & $\begin{array}{l}\text { n-hexane: dichloromethane: } \\
(80: 20 \mathrm{v} / \mathrm{v})\end{array}$ & Comp. 2 \\
\hline \multirow[t]{2}{*}{2} & 1.45 & $\begin{array}{l}\text { dichloromethane: ethyl acetate } \\
(70: 30 \mathrm{v} / \mathrm{v})\end{array}$ & Comp. 3 \\
\hline & & $\begin{array}{l}\text { dichloromethane: ethyl acetate } \\
(50: 50 \mathrm{v} / \mathrm{v})\end{array}$ & Comp. 4 \\
\hline \multirow[t]{3}{*}{3} & 1.9 & $\begin{array}{l}\text { ethyl acetate: methanol } \\
(95: 5 \mathrm{v} / \mathrm{v})\end{array}$ & Comp. 5 \\
\hline & & $\begin{array}{l}\text { ethyl acetate: methanol } \\
(90: 10 \mathrm{v} / \mathrm{v})\end{array}$ & Comp. 6 \\
\hline & & $\begin{array}{l}\text { ethyl acetate : methanol } \\
(85: 15 \mathrm{v} / \mathrm{v})\end{array}$ & Comp. 7 \\
\hline \multirow[t]{3}{*}{4} & 1.65 & $\begin{array}{l}\text { ethyl acetate : methanol } \\
(75: 25 \mathrm{v} / \mathrm{v})\end{array}$ & Comp. 8 \\
\hline & & $\begin{array}{l}\text { ethyl acetate: methanol } \\
(60: 40: 10 \mathrm{v} / \mathrm{v})\end{array}$ & Comp. 9 \\
\hline & & Further elution with methanol & Comp. 10 \\
\hline
\end{tabular}

process. The extract was concentrated under reduced pressure to give $39 \mathrm{~g}$ of the crude extract and the extract was subjected to preliminary phytochemical analysis to detect the main phytoconstituents and all the phytochemical tests were carried out according to that described by Yadav and Agarwala (2011).

\section{Phytochemical Characterization of $F$. vasta aerial parts} methanol $80 \%$ extract

All the isolated compounds were purified on Sephadex LH-20 column using methanol and different mixtures of methanol and distilled water.

\section{General method for acid hydrolysis of flavonoid glycosides}

$5 \mathrm{mg}$ of each flavonoid glycoside 8, 9 and 10 in $5 \mathrm{ml} \mathrm{10 \%}$ $\mathrm{HCl}$ was heated for $5 \mathrm{~h}$. The agly cones were extracted with EtOAc and identified by co-TLC with authentic standards. The sugars in the aqueous layer were identified by copaper chromatography (co-PC) with authentic markers on Whatman No. 1 sheets in solvent system $(n-\mathrm{BuOH}-\mathrm{AcOH}-$ $\mathrm{H}_{2} \mathrm{O}$ 4:1:5 upper layer).

\section{Cytotoxicity assay}

Vero cells (epithelial cells from kidney of Cercopithecus aethiops) were cultured in 96-well microplates, and the monolayers were incubated for $72 \mathrm{~h}$ at $37^{\circ} \mathrm{C}$ and $5 \% \mathrm{CO}_{2}$ with DMEM containing $5 \%$ fetal bovine serum (FBS) and $1 \%$ dimethyl sulfoxide (DMSO), penicillin G (100 IU/ml), enrofloxacin $(10 \mu \mathrm{g} / \mathrm{ml})$ and amphotericin B $(1.25 \mu \mathrm{g} / \mathrm{ml})$ with 2-fold serial dilutions of the extract at different concentrations, ranging from from 3.8 to $1900 \mu \mathrm{g} / \mathrm{ml}$ to methanol $80 \%$ of $F$. vasta methanol extract. For this, 3 repetitions of 8 wells were used for the evaluation of extract dilution. The in vitro toxicity of methanol $80 \%$ of $F$. vasta extract which was sterilized by filtration through PVDF membranes (pore size $0.22 \mu \mathrm{m}$ ), was determined by quantifying the viable cells using 3-[4,5-dimethyl-thiazol2-yl]-2,5-diphenyltetrazolium bromide (MTT), which is converted into a purple formazan by mitochondrial dehydrogenases (Denizot and Lang, 1986). Fifty percent cytotoxic concentration $\left(\mathrm{CC}_{50}\right)$ was defined as the extract

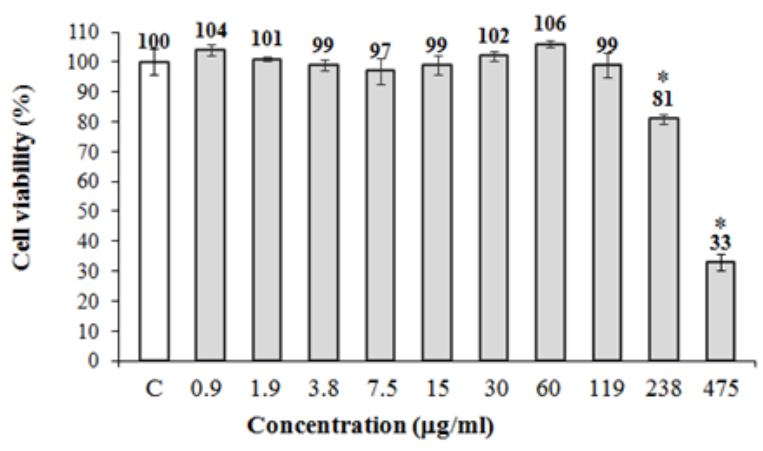

Figure 1: Evaluation of cytotoxicity of F. vasta methanol extract in Vero cells after $72 \mathrm{~h}$ of incubation at $37^{\circ} \mathrm{C}$ and $5 \% \mathrm{CO}_{2}$ by the MTT assay. Control: without extract treatment. Bars represent means, with vertical lines indicating standard deviations, $\mathrm{n}=3$, ${ }^{*} \mathrm{P}<0.01$.

concentration which could reduce by $50 \%$ the number of viable cells, when compared with a control without it, and it was calculated by regression analysis of the doseresponse curves.

\section{Antiviral assay}

The screening of the antiviral activity of $F$. vasta methanol extract against HSV-1 was carried out on confluent monolayers of Vero cells in 96-well microplates by adding the highest non-toxic concentration determined by the MTT method $(119 \mu \mathrm{g} / \mathrm{mL})$ during the HSV-1 (100xTCID 50$)$ adsorption step and after adsorption in the same concentrations within the maintenance medium (DMEM with 5\% FBS and $1 \%$ DMSO added by antibiotics and antifungal). The viral replication was performed for $72 \mathrm{~h}$ at $37^{\circ} \mathrm{C}$ and $5 \% \mathrm{CO}_{2}$ incubation. The inhibition of the HSV-1 replication was related to the absence of any viral induced cytopathic effect at the highest non-toxic concentration of the extract, evaluated by observation of the monolayers under microscope, when compared with a control without it. Thus this test aimed to verify a decrease in the virus titer obtained by the endpoint titration method (Reed and Müench, 1938) due to the plant extract.

\section{Antibacterial assay}

The antibacterial activity of $F$. vasta methanol extract was evaluated against Staphylococcus aureus (ATCC 25923) Staphylococcus epidermidis (ATCC 12228), Escherichia coli (ATCC 25922) and Pseudomonas aeruginosa (ATCC 27853) strains by the disk-diffusion method. Briefly, sterilized filter paper disks of $6 \mathrm{~mm}$ diameter were impregnated with $10 \mu \mathrm{L}$ of the control substances (1\% DMSO, $0.05 \%$ enrofloxacine or $20 \%$ chlorhexidine) or with extract solution $(119 \mu \mathrm{g} / \mathrm{mL}$ in $1 \%$ DMSO) previously sterilized by filtration on polyvinylidene difluoride membranes with pore diameter of $0.22 \mu \mathrm{m}$. The impregnated disks $(n=3)$ containing $1.19 \mu \mathrm{g}$ of the extract were positioned on the top of the Petri dishes containing $25 \mathrm{~mL}$ of MuellerHinton Agar previously seeded with a bacterial suspension adjusted to the 0.5 degree of the McFarland turbidity scale, which corresponds to a concentration of $1.5 \times 10^{8}$ $\mathrm{CFU} / \mathrm{mL}$. The plates were incubated at $35^{\circ} \mathrm{C}$ for $24 \mathrm{~h}$ and after that, the diameter of the zone of inhibition of the bacterial growth around the discs were determined. 
Table 2: Spectroscopic analyses of the isolated compounds of $F$. vasta methanol extract.

\begin{tabular}{|c|c|c|c|c|c|c|}
\hline $\begin{array}{l}\text { Compound } \\
\text { (No.) }\end{array}$ & $\begin{array}{l}\text { Amount } \\
\text { recovered } \\
(\mathrm{mg})\end{array}$ & $\begin{array}{c}\text { Physical } \\
\text { state }\end{array}$ & 1'H-NMR data & ${ }^{13} \mathrm{C}-\mathrm{NMR}$ data & UV data & MS data \\
\hline $\begin{array}{c}\beta \text {-sitosterol } \\
\text { (1) }\end{array}$ & 12 & $\begin{array}{l}\text { White } \\
\text { needles }\end{array}$ & $\begin{array}{l}{ }^{1} \mathrm{H}-\mathrm{NMR}\left(400 \mathrm{MHz}, \mathrm{CDCl}_{3}\right): \delta \\
5.37(\mathrm{IH}, \mathrm{m}, \mathrm{H}-6), 3.52(\mathrm{IH}, \mathrm{m}, \mathrm{H}- \\
\text { 3) }, 1.09(3 \mathrm{H}, \mathrm{s}, \mathrm{CH} 3-19), 0.98 \\
(3 \mathrm{H}, \mathrm{d}, \mathrm{J}=6.5, \mathrm{CH} 3-21), 0.92(3 \mathrm{H}, \\
\mathrm{t}, \mathrm{J}=7.4, \mathrm{CH}-29), 0.85(3 \mathrm{H}, \mathrm{d}, \mathrm{J}= \\
6.7 \mathrm{~Hz}, \mathrm{CH}-26), 0.81(3 \mathrm{H}, \mathrm{d}, \mathrm{J}= \\
6.7 \mathrm{~Hz}, \mathrm{CH}-27), 0.75(3 \mathrm{H}, \mathrm{s}, \\
\mathrm{CH} 3-18)\end{array}$ & $\begin{array}{l}{ }^{13} \mathrm{C}-\mathrm{NMR}\left(100 \mathrm{MHz}, \mathrm{CDCl}_{3}\right): \delta \\
140.4(\mathrm{C}-5), 121.5(\mathrm{C}-6), 71.6 \text { (C-3), } \\
57.2(\mathrm{C}-17), 56.4(\mathrm{C}-14), 50.3(\mathrm{C}-9), \\
46.3(\mathrm{C}-24), 42,8(\mathrm{C}-13,4), 39.8(\mathrm{C}- \\
12), 37.6(\mathrm{C}-1), 36.7(\mathrm{C}-10), 35.9 \\
(\mathrm{C}-20), 34.2(\mathrm{C}-22), 31.7(\mathrm{C}-8,7), \\
31.4(\mathrm{C}-2), 29.2(\mathrm{C}-25), 28.4(\mathrm{C}-16), \\
26.2(\mathrm{C}-23), 24.5(\mathrm{C}-15), 23.4(\mathrm{C}- \\
28), 21.1(\mathrm{C}-11), 19.8(\mathrm{C}-26), 19.5 \\
(\mathrm{C}-19), 19.2(\mathrm{C}-27), 18.6(\mathrm{C}-21) .\end{array}$ & & \\
\hline $\begin{array}{c}\text { Stigmasterol } \\
\text { (2) }\end{array}$ & 15 & $\begin{array}{l}\text { white } \\
\text { needle } \\
\text { crystals }\end{array}$ & $\begin{array}{l}{ }^{1} \mathrm{H}-\mathrm{NMR}\left(400 \mathrm{MHz}, \mathrm{CDCl}_{3}\right): \delta \\
5.32(\mathrm{IH}, \mathrm{m}, \mathrm{H}-6), 5.11(1 \mathrm{H}, \mathrm{dd}, J= \\
14.2,8.2 \mathrm{~Hz}, \mathrm{H}-22), 5.04(1 \mathrm{H}, \mathrm{dd}, \\
\mathrm{J}=14.2,8.2 \mathrm{~Hz}, \mathrm{H}-23), 3.54(\mathrm{IH}, \\
\mathrm{m}, \mathrm{H}-3), 1.04\left(3 \mathrm{H}, \mathrm{s}, \mathrm{CH}_{3}-10\right), 0.9 \\
\left(3 \mathrm{H}, \mathrm{d}, J=6.5, \mathrm{CH}_{3}-20\right), 0.84(3 \mathrm{H}, \\
\left.\mathrm{d}, \mathrm{J}=7.4, \mathrm{CH}_{3}-27\right), 0.82(3 \mathrm{H}, \mathrm{d}, J= \\
\left.7.4, \mathrm{CH}_{3}-26\right), 0.68\left(3 \mathrm{H}, \mathrm{s}, \mathrm{CH}_{3}-\right. \\
13) .\end{array}$ & $\begin{array}{l}{ }^{13} \mathrm{C}-\mathrm{NMR}\left(100 \mathrm{MHz}, \mathrm{CDCl}_{3}\right): \delta \\
140.4(\mathrm{C}-5), 121.5(\mathrm{C}-6), 71.6(\mathrm{C}-3), \\
57.2(\mathrm{C}-17), 56.4(\mathrm{C}-14), 50.3(\mathrm{C}-9), \\
46.3(\mathrm{C}-24), 42,8(\mathrm{C}-13,4), 39.8(\mathrm{C}- \\
12), 37.6(\mathrm{C}-1), 36.7(\mathrm{C}-10), 35.9(\mathrm{C}- \\
20), 34.2(\mathrm{C}-22), 31.7(\mathrm{C}-8,7), 31.4 \\
(\mathrm{C}-2), 29.2(\mathrm{C}-25), 28.4(\mathrm{C}-16), 26.2 \\
(\mathrm{C}-23), 24.5(\mathrm{C}-15), 23.4(\mathrm{C}-28), \\
21.1(\mathrm{C}-11), 19.8(\mathrm{C}-26), 19.5(\mathrm{C}- \\
19), 19.2(\mathrm{C}-27), 18.6(\mathrm{C}-21)\end{array}$ & & \\
\hline Lupeol (3) & 14 & $\begin{array}{l}\text { White } \\
\text { powder }\end{array}$ & $\begin{array}{l}{ }^{1} \mathrm{H}-\mathrm{NMR}\left(\mathrm{CDCl}_{3}, 400 \mathrm{MHz}\right): \delta \\
0.75,0.8,0.85,0.96,0.98,1.08, \\
1.75 \text { (each } 3 \mathrm{H}, \mathrm{s}), 3.25(1 \mathrm{H}, \mathrm{dd}, J \\
=5.6,10.8 \mathrm{~Hz}, \mathrm{H}-3), 4.58(1 \mathrm{H}, \mathrm{s}, \\
\mathrm{H}-29 \mathrm{a}), 4.68(1 \mathrm{H}, \mathrm{s}, \mathrm{H}-29 \mathrm{~b}) .\end{array}$ & $\begin{array}{l}{ }^{13} \mathrm{CNMR}(\mathrm{CDCl}, 100 \mathrm{MHz}): \delta 151.4 \\
(\mathrm{C}-20), 108.7(\mathrm{C}-29), 78.6(\mathrm{C}-3), \\
55.8 \text { (C-5), } 50.7(\mathrm{C}-9), 48.7(\mathrm{C}-18), \\
48.4(\mathrm{C}-19), 43.2(\mathrm{C}-17), 43.2(\mathrm{C}- \\
14), 40.8(\mathrm{C}-8), 39.7(\mathrm{C}-22), 38.7 \\
(\mathrm{C}-4), 38.5(\mathrm{C}-1), 38.7(\mathrm{C}-13), 37.6 \\
(\mathrm{C}-10), 35.7(\mathrm{C}-16), 34.5(\mathrm{C}-7), \\
29.4(\mathrm{C}-21), 28.4(\mathrm{C}-23), 27.6(\mathrm{C}-2), \\
27.6(\mathrm{C}-15), 25.4(\mathrm{C}-12), 21.4(\mathrm{C}- \\
11), 19.4(\mathrm{C}-30), 18.7(\mathrm{C}-6), 18.4 \\
(\mathrm{C}-28), 16.5(\mathrm{C}-25), 16.2(\mathrm{C}-26), \\
15.7(\mathrm{C}-24), 15.2(\mathrm{C}-27) .\end{array}$ & & \\
\hline $\begin{array}{l}\text { Ursolic acid } \\
(4)\end{array}$ & 10 & $\begin{array}{l}\text { White } \\
\text { powder }\end{array}$ & $\begin{array}{l}{ }^{1} \mathrm{H}-\mathrm{NMR}\left(\mathrm{CDCl}_{3}, 400 \mathrm{MHz}\right): \delta \\
5.26(\mathrm{IH}, \mathrm{t}, J=3.5, \mathrm{H}-12), 3.17(1 \mathrm{H}, \\
\mathrm{dd}, J=10,4.2 \mathrm{~Hz}, \mathrm{H}-3), 2.15(1 \mathrm{H}, \\
\mathrm{d}, J=11.5 \mathrm{~Hz}, \mathrm{H}-18), 1.92(1 \mathrm{H}, \mathrm{dd}, \\
J=12.8,4.2 \mathrm{~Hz}, \mathrm{Hb}-22), 1.12(1 \mathrm{H}, \\
\text { m, Ha-22), } 1.22(3 \mathrm{H}, \mathrm{s}, \mathrm{Me}-23), \\
0.94(3 \mathrm{H}, \mathrm{s}, \mathrm{Me}-24), 0.75(3 \mathrm{H}, \mathrm{s}, \\
\mathrm{Me}-25), 1.04(3 \mathrm{H}, \mathrm{s}, \mathrm{Me}-26), 1.12 \\
(3 \mathrm{H}, \mathrm{s}, \mathrm{Me}-27), 0.92(3 \mathrm{H}, \mathrm{d}, J=6.4 \\
\mathrm{Hz}, \mathrm{Me}-29), 0.89(3 \mathrm{H}, \mathrm{d}, J=5.8 \mathrm{~Hz}, \\
\text { Me-30) }\end{array}$ & & & $\begin{array}{l}(+) \text { ESI- } \\
\text { MS:m/z } \\
455[\mathrm{M}- \\
\mathrm{H}]^{+}\end{array}$ \\
\hline $\begin{array}{l}\text { Naringnin } \\
\text { (5) }\end{array}$ & 14 & $\begin{array}{l}\text { Yellow } \\
\text { needles }\end{array}$ & $\begin{array}{l}{ }^{1} \mathrm{H}-\mathrm{NMR}\left(300 \mathrm{MHz}, \mathrm{CD}_{3} \mathrm{OD}\right) \delta \\
2.95(1 \mathrm{H}, \mathrm{dd}, \mathrm{J}=1.7,9.5 \mathrm{~Hz}, \mathrm{H}- \\
3 \alpha), 3.02(1 \mathrm{H}, \mathrm{dd}, \mathrm{J}=9.5,12.4 \\
\mathrm{Hz}, \mathrm{H}-3 \beta), 5.15(1 \mathrm{H}, \mathrm{dd}, \mathrm{J}=1.7, \\
9.5 \mathrm{~Hz}, \mathrm{H}-2 \alpha), 5.78(2 \mathrm{H}, \mathrm{s}, \mathrm{H}-6, \\
8), 6.72\left(2 \mathrm{H}, \mathrm{d}, \mathrm{J}=6.5 \mathrm{~Hz}, \mathrm{H}-3^{\prime},\right. \\
\left.5^{\prime}\right), 7.28\left(2 \mathrm{H}, \mathrm{d}, \mathrm{J}=6.5 \mathrm{~Hz}, \mathrm{H}-2^{\prime},\right. \\
\left.6^{\prime}\right) .\end{array}$ & & $\begin{array}{l}\text { UV } \lambda \mathrm{max} \\
(\mathrm{MeOH}): 289, \\
\text { 324sh, } \\
(\mathrm{NaOMe}): 246, \\
\text { 265sh, } 328 \\
(\mathrm{AlCl} 3): 312, \\
374\left(\mathrm{AlCl}_{3} /\right. \\
\mathrm{HCl} \text { 312, 375, } \\
(\mathrm{NaOAc}): 294 \\
\text { sh, 325 } \\
\left(\mathrm{NaOAc} / \mathrm{H}_{3} \mathrm{~B}\right. \\
\left.\mathrm{O}_{3}\right): 290, \\
\text { 324sh, } 332 \text { sh. }\end{array}$ & $\begin{array}{l}\text { EI-MS: } \\
\text { m/z } 272 .\end{array}$ \\
\hline Luteolin (6) & 8 & $\begin{array}{l}\text { Yellow } \\
\text { powder }\end{array}$ & $\begin{array}{l}{ }^{1} \mathrm{H}-\mathrm{NMR}(\mathrm{DMSO}-\mathrm{d} 6,400 \mathrm{MHz}) \\
\delta \mathrm{ppm} 12.9(1 \mathrm{H}, \mathrm{s}, 5-\mathrm{OH}), 7.4 \\
\left(1 \mathrm{H}, \mathrm{d}, J=8 \mathrm{~Hz},, \mathrm{H}-6^{\prime}\right), 7.38 \mathrm{(1 \textrm {H }}, \\
\left.\mathrm{d}, J=2 \mathrm{~Hz}, \mathrm{H}-2^{\prime}\right), 6.85(1 \mathrm{H}, \mathrm{d}, J= \\
\left.8 \mathrm{~Hz}, \mathrm{H}-5^{\prime}\right), 6.6(1 \mathrm{H} \mathrm{s}, \mathrm{H}-3), 6.4 \\
(1 \mathrm{H}, \mathrm{d}, J=2 \mathrm{~Hz}, \mathrm{H}-8), 6.15(1 \mathrm{H}, \\
\text { d, J=2 Hz, H-6). }\end{array}$ & & & $\begin{array}{l}\text { EI-MS: } \\
\text { m/z } 286\end{array}$ \\
\hline
\end{tabular}


Table 2: Cont.

\begin{tabular}{|c|c|c|c|c|c|c|}
\hline $\begin{array}{l}\text { Compound } \\
\text { (No.) }\end{array}$ & $\begin{array}{c}\text { Amount } \\
\text { recovered } \\
(\mathrm{mg})\end{array}$ & $\begin{array}{c}\text { Physical } \\
\text { state }\end{array}$ & ${ }^{1} \mathrm{H}-\mathrm{NMR}$ data & ${ }^{13}$ C-NMR data & UV data & MS data \\
\hline $\begin{array}{l}\text { Quercetin } \\
\text { (7) }\end{array}$ & 11 & $\begin{array}{l}\text { Yellow } \\
\text { powder }\end{array}$ & & & $\begin{array}{l}\mathrm{UV} \lambda \mathrm{max} \\
(\mathrm{MeOH}): 255, \\
267,371 ; \\
(\mathrm{NaOMe}): \\
270,320,420 ; \\
\left(\mathrm{AlCl}_{3}\right): 270, \\
455 ; \\
\left(\mathrm{AlCl}_{3} / \mathrm{HCl}\right): \\
264,303 \mathrm{sh}, \\
315 \mathrm{sh}, 428 ; \\
(\mathrm{NaOAc}): 257, \\
274,318,383 ; \\
\left(\mathrm{NaOAc} / \mathrm{H}_{3} \mathrm{~B}\right. \\
\left.\mathrm{O}_{3}\right): 259,387\end{array}$ & $\begin{array}{l}\text { EI-MS: } \\
\mathrm{m} / \mathrm{z} 302\end{array}$ \\
\hline Vitexin (8) & 15 & $\begin{array}{l}\text { yellow } \\
\text { amor- } \\
\text { phous } \\
\text { powder }\end{array}$ & $\begin{array}{l}{ }^{1} \mathrm{H}-\mathrm{NMR}\left(\mathrm{DMSO}-\mathrm{d}_{6}, 400 \mathrm{MHz}\right): \\
\delta 8.04\left(\mathrm{~d}, J=8.5 \mathrm{~Hz}, 2 \mathrm{H}, \mathrm{H}-2^{\prime}, 6^{\prime}\right) \\
6.88\left(\mathrm{~d}, J=8.5 \mathrm{~Hz}, 2 \mathrm{H}, \mathrm{H}-3^{\prime}, 5^{\prime}\right), \\
6.42(\mathrm{~s}, 1 \mathrm{H}, \mathrm{H}-3), 6.74(\mathrm{~s}, 1 \mathrm{H}, \mathrm{H}- \\
6), 4.65\left(\mathrm{~d}, J=9.6 \mathrm{~Hz}, 1 \mathrm{H}, \mathrm{H}-1^{\prime \prime}\right) .\end{array}$ & & $\begin{array}{l}\text { UV } \lambda \text { max } \\
(\mathrm{MeOH}): 269, \\
331 ; \\
(\mathrm{NaOMe}): \\
279,325(\mathrm{sh}), \\
391 ;\left(\mathrm{AlCl}_{3}\right): \\
276,303(\mathrm{sh}), \\
346,382 ; \\
\left(\mathrm{AlCl}_{3} / \mathrm{HCl}\right): \\
277 ; 303,343, \\
380(\mathrm{NaOAc}): \\
278,387 \\
\left(\mathrm{NaOAc} / \mathrm{H}_{3} \mathrm{~B}\right. \\
\left.\mathrm{O}_{3}\right): 270,319, \\
346\end{array}$ & $\begin{array}{l}\text { ESI-MS } \\
m / z: 433 \\
{[\mathrm{M}+\mathrm{H}]^{+}}\end{array}$ \\
\hline $\begin{array}{l}\text { Quercetin 3- } \\
O-\beta \text { - } \\
\text { glucoside (9) }\end{array}$ & 12 & $\begin{array}{l}\text { Yellow } \\
\text { crystals }\end{array}$ & $\begin{array}{l}{ }^{1} \mathrm{H}-\mathrm{NMR}(\mathrm{DMSO}-\mathrm{d} 6,400 \mathrm{MHz}): \delta \\
7.78\left(1 \mathrm{H}, \mathrm{dd}, J=2,8.5 \mathrm{~Hz}, \mathrm{H}-6{ }^{\prime}\right), \\
7.54\left(1 \mathrm{H}, \mathrm{d}, J=2 \mathrm{~Hz}, \mathrm{H}-2^{\prime}\right), 6.82 \\
\left(1 \mathrm{H}, \mathrm{d}, J=8.5 \mathrm{~Hz}, \mathrm{H}-5^{\prime}\right), \mathrm{d} 6.42 \\
(1 \mathrm{H}, \mathrm{d}, J=2 \mathrm{~Hz}, \mathrm{H}-8), 6.24(1 \\
\mathrm{H}, \mathrm{d}, J=2 \mathrm{~Hz}, \mathrm{H}-6), 5.5(1 \mathrm{H}, \mathrm{d}, \\
\left.J=7.5 \mathrm{~Hz}, \mathrm{H}-1^{\prime}\right) .\end{array}$ & & & $\begin{array}{l}\text { (-) ESI- } \\
\text { MS: m/z } \\
463 \\
{[\mathrm{M}-\mathrm{H}]^{-}}\end{array}$ \\
\hline $\begin{array}{l}\text { Quercetin 3- } \\
\text { O-rutinoside } \\
\text { (Rutin) (10) }\end{array}$ & 18 & $\begin{array}{l}\text { Yellow } \\
\text { powder }\end{array}$ & $\begin{array}{l}{ }^{1} \mathrm{H}-\mathrm{NMR}(400 \mathrm{MHz}, \mathrm{DMSO}-\mathrm{d} 6): \\
\delta \mathrm{ppm} 7.54\left(2 \mathrm{H}, \mathrm{m}, \mathrm{H}-2^{\prime} / 6^{\prime}\right), 6.85 \\
\left(1 \mathrm{H}, \mathrm{d}, J=9 \mathrm{~Hz}, \mathrm{H}-5^{\prime}\right), 6.38(1 \mathrm{H}, \\
\mathrm{d}, J=2.5 \mathrm{~Hz}, \mathrm{H}-8), 6.19(1 \mathrm{H}, J= \\
2.5 \mathrm{~Hz}, \mathrm{H}-6), 5.35(1 \mathrm{H}, \mathrm{d}, J=7.5 \\
\left.\mathrm{Hz}, \mathrm{H}-1^{\prime \prime}\right), 4.39\left(1 \mathrm{H}, \mathrm{s}, \mathrm{H}-1^{\prime \prime \prime}\right), \\
3.90-3.20(\mathrm{~m}, \text { remaining sugar } \\
\text { protons), } 0.99(3 \mathrm{H}, \mathrm{d}, \mathrm{J}=6 \mathrm{~Hz}, \\
\left.\mathrm{H}^{\prime} 6^{\prime \prime \prime}\right) .\end{array}$ & $\begin{array}{l}{ }^{13} \mathrm{C}-\mathrm{NMR}(\mathrm{DMSO}-\mathrm{d} 6,100 \mathrm{MHz}): \delta \\
\text { ppm 177.85(C-4), 164.70(C-7), } \\
\text { 161.68(C-5), 157.14(C-2), 156.95(C- } \\
\text { 9), 148.92(C-4'), 145.25(C-3'), } \\
133.76(\mathrm{C}-3), 122.12\left(\mathrm{C}-6^{\prime}\right), \\
121.66\left(\mathrm{C}-1^{\prime}\right), 116.73\left(\mathrm{C}-2^{\prime}\right), \\
115.72\left(\mathrm{C}-5^{\prime}\right), 104.41(\mathrm{C}-10), \\
101.66\left(\mathrm{C}-1^{\prime \prime \prime}\right), 101.23\left(\mathrm{C}-1^{\prime \prime}\right), 99.24 \\
(\mathrm{C}-6), 94.16(\mathrm{C}-8), 74.58\left(\mathrm{C}-3^{\prime \prime}\right), \\
72.33\left(\mathrm{C}-5^{\prime \prime}\right), 72.2\left(\mathrm{C}-4^{\prime \prime \prime}\right), 71.05(\mathrm{C}- \\
\left.2^{\prime \prime}\right), 70.8\left(\mathrm{C}-2^{\prime \prime \prime}\right), 70.87(\mathrm{C}- \\
\left.3^{\prime \prime \prime}\right), 70.49\left(\mathrm{C}-4^{\prime \prime}\right), 63.74(\mathrm{C}- \\
\left.6^{\prime \prime}\right), 18.19\left(\mathrm{C}-6^{\prime \prime \prime}\right) .\end{array}$ & $\begin{array}{l}\text { UV } \lambda \text { max } \\
(\mathrm{MeOH}): 258, \\
\text { 269, 361; } \\
(\mathrm{NaOMe}): \\
276,322,416 ; \\
\left(\mathrm{AlCl}_{3}\right): 232, \\
276,302,366 ; \\
\left(\mathrm{AlCl}_{3} / \mathrm{HCl}\right): \\
232,276,302, \\
366 ;(\mathrm{NaO} \mathrm{Ac}): \\
284,306,381 ; \\
(\mathrm{NaO} \mathrm{Ac} / \mathrm{H} 3 \mathrm{~B} \\
\left.\mathrm{O}_{3}\right): 261,312, \\
376\end{array}$ & \\
\hline
\end{tabular}

Table 3: Phytochemical Analysis of F. vasta aerial parts methanol $80 \%$ extract.

\begin{tabular}{lc}
\hline Constituents & Methanol extract \\
\hline Triterpenes and /or Sterols & + \\
Carbohydrates and/or glycosides & + \\
Flavonoids & + \\
Coumarins & + \\
Alkaloids and/or nitrogenous compounds & + \\
Tannins & + \\
Saponins & - \\
\hline
\end{tabular}

$(+)$ presence of constituents, (-) absence of constituents

\section{Statistical analysis}

All biological experiments were statistically expressed as mean + standard deviation, and analyzed by Student's $t$ test with $P<0.01$. Variables exceeding the upper quantification limit were considered statistically significant. 


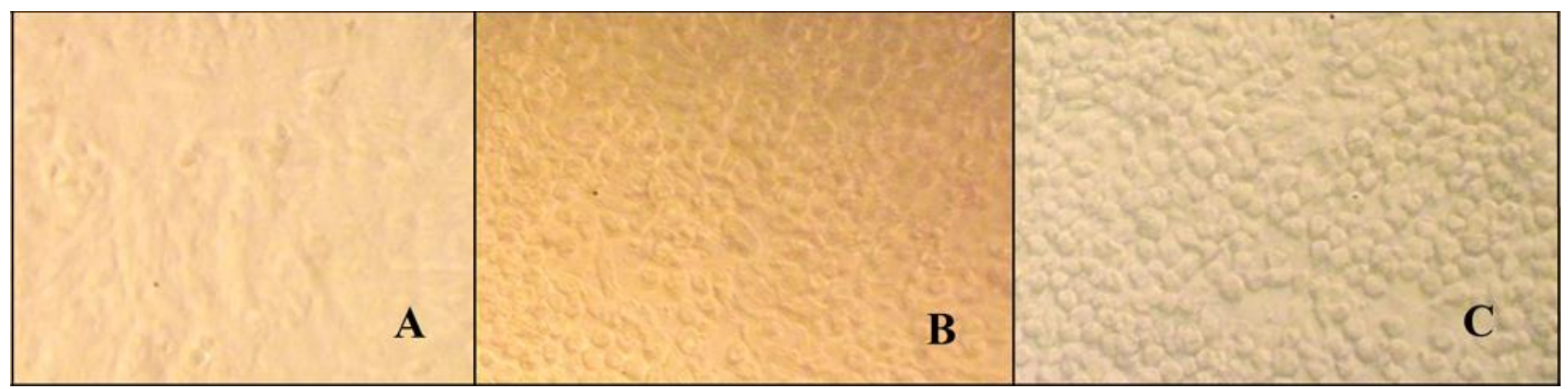

Figure 2: Screening of the anti-HSV-1 activity in Vero cells after $72 \mathrm{~h}$ of incubation at $37^{\circ} \mathrm{C}$ and $5 \% \mathrm{CO}_{2}$ in 96 -well microplates.

(A): negative control, DMEM 5\% FBS; (B): positive control, 100xTCID50 of HSV-1; (C): 100xTCID50 of HSV-1 + methanol 80\% extract of $F$. vasta aerial parts at $119 \mu \mathrm{g} / \mathrm{mL}$. Magnification of 200x.

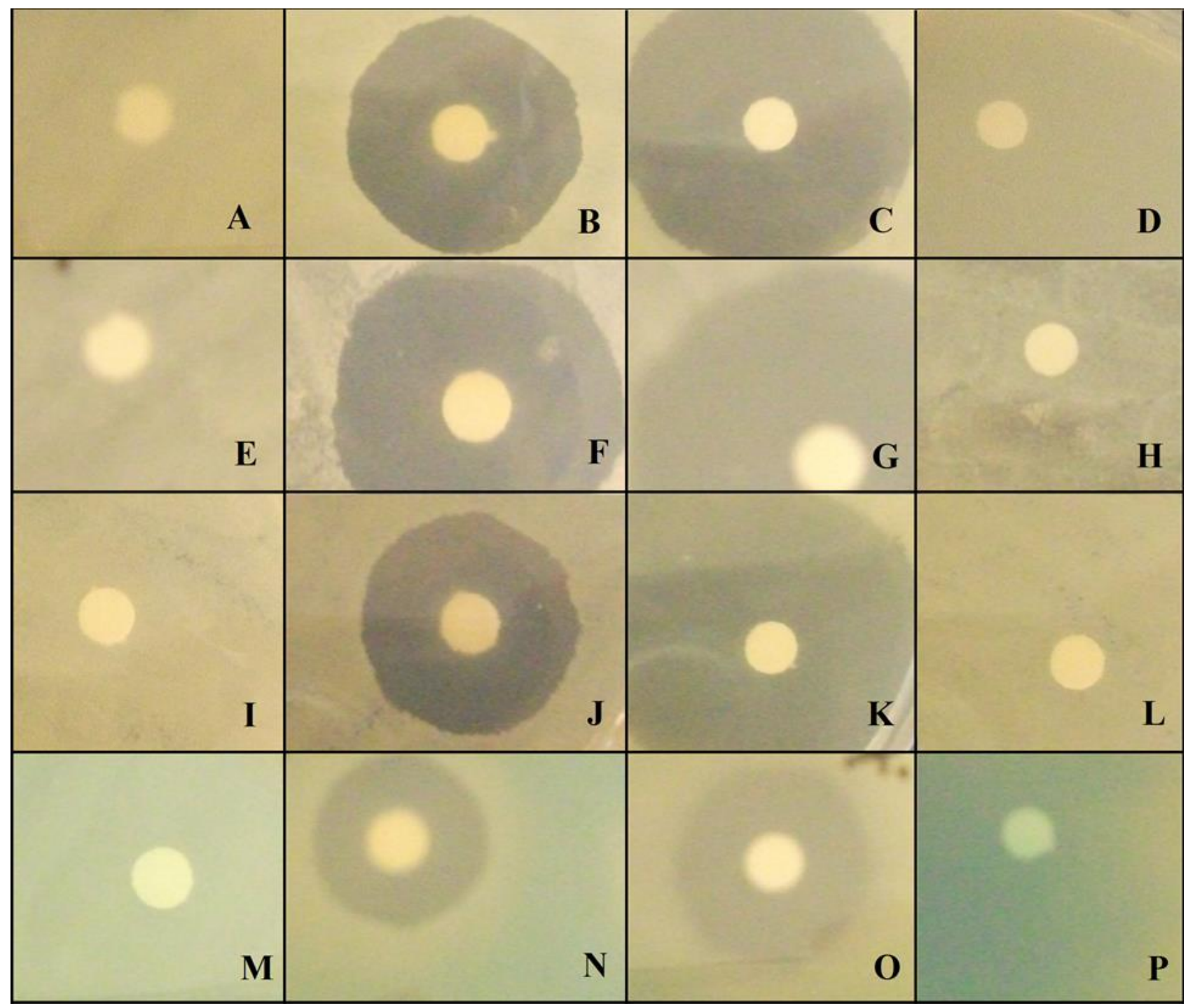

Figure 3: Screening of the antibacterial activity of the methanol extract of $F$. vasta by the disk-diffusion method after $24 \mathrm{~h}$ of incubation at $37^{\circ} \mathrm{C}$. (A): S. aureus and $1 \%$ DMSO; (B): S. aureus and $20 \%$ chlorhexidine; (C): S. aureus and $0.05 \%$ enrofloxacine; (D): S. aureus and extract at $119 \mu \mathrm{g} / \mathrm{mL}$; (E): S. epidermidis and 1\% DMSO; (F): S. epidermidis and 20\% chlorhexidine; (G): S. epidermidis and $0.05 \%$ enrofloxacine; $(\mathrm{H})$ : S. epidermidis and extract at $119 \mu \mathrm{g} / \mathrm{mL} ;(\mathrm{I})$ : E. coli and 1\% DMSO; (J): E. coli and $20 \%$ chlorhexidine; (K): E. coli and $0.05 \%$ enrofloxacine; (L): E. coli and extract at $119 \mu \mathrm{g} / \mathrm{mL}$; (M): P. aeruginosa and 1\% DMSO; (N): P. aeruginosa and 20\% chlorhexidine; (O): P. aeruginosa and 0.05\% enrofloxacine; (P): P. aeruginosa and extract at $119 \mu \mathrm{g} / \mathrm{mL}$. 


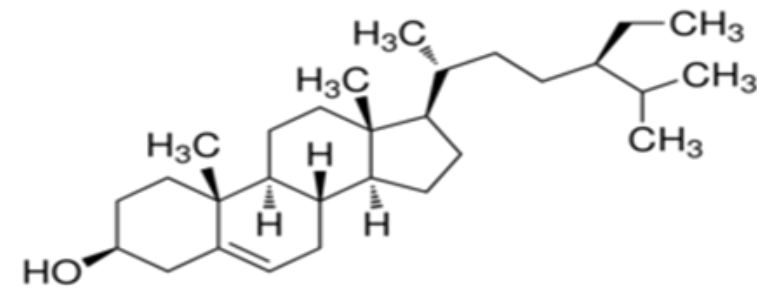

$\beta$-sitosterol (1)

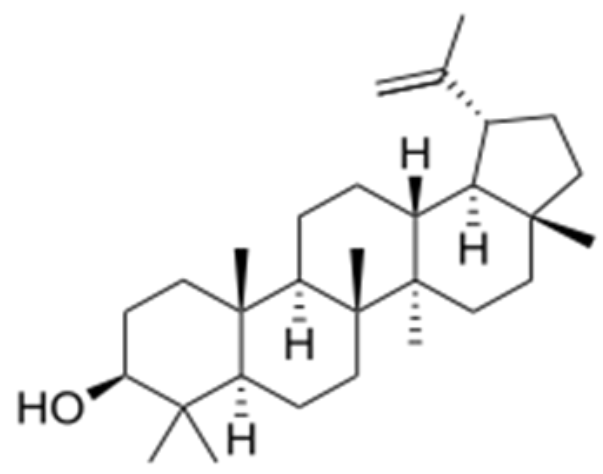

Lupeol (3)<smiles>O=C1C[C@H](c2ccc(O)cc2)Oc2cc(O)cc(O)c21</smiles>

Naringenin (5)<smiles>O=c1c(O)c(-c2ccc(O)c(O)c2)oc2cc(O)cc(O)c12</smiles>

Quercetin (7)<smiles>[R]Oc1c(-c2ccc(O)c(O)c2)oc2cc(O)cc(O)c2c1=O</smiles>

Quercetin 3-O- $\beta$-glucoside (R=glucose) (9)

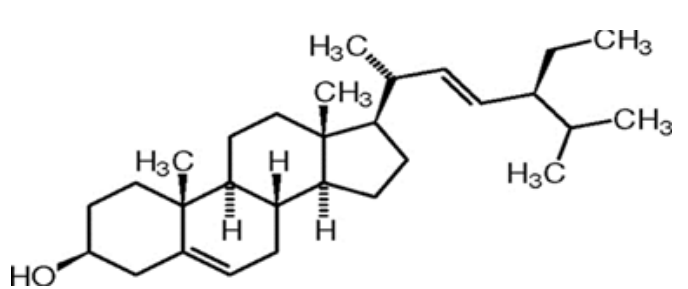

Stigmasterol (2)

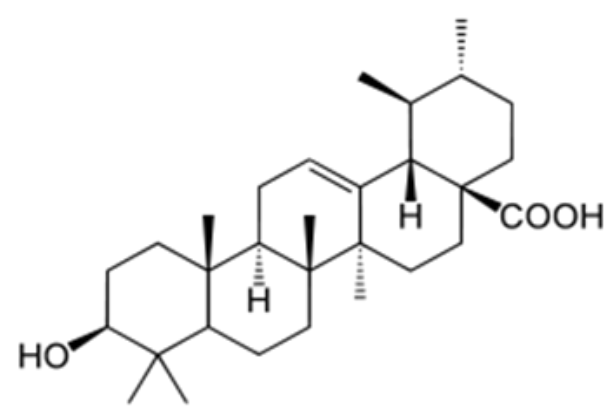

Ursolic acid (4)<smiles>O=c1cc(-c2ccc(O)c(O)c2)oc2cc(O)cc(O)c12</smiles><smiles>[R]c1c(O)cc(O)c2c(=O)cc(-c3ccc(O)cc3)oc12</smiles>

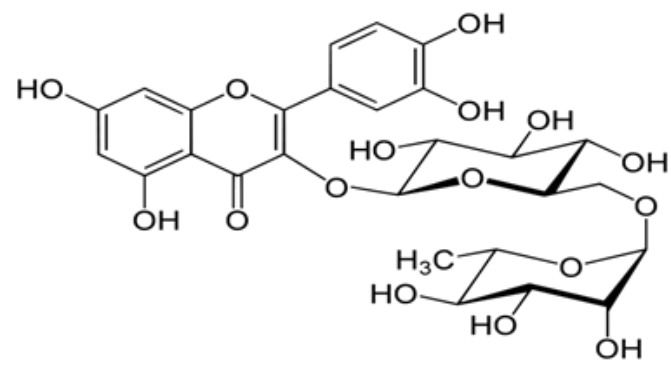

Rutin (10)

Figure 4: Chemical structures of compounds isolated from F. vasta methanol extract. 


\section{RESULTS AND DISCUSSION}

The present investigation evaluated the cytotoxicity, antiviral and antibacterial activities of $F$. vasta aerial parts methanol extract in vitro, determined the main phytoconstituents of the extract which are carbohydrates, tannins, flavonoids, coumarins, alkaloids and triterpenes and also detected the bioactive phytoconstituents of $F$. vasta methanol extract which are $\beta$-sitosterol, stigmasterol, lupeol, ursolic acid, naringenin, luteolin, quercetin, vitexin, quercetin 3-O- $\beta$-glu coside and rutin.

\section{Evaluation of cytotoxicity, antiviral and antibacterial activities}

$F$. vasta methanol extract did not decrease the percentage of viable cells up to $119 \mu \mathrm{g} / \mathrm{mL}(P>0.01)$, presenting a C $C_{50}$ $=389 \mu \mathrm{g} / \mathrm{mL}$ in Vero cells. This value is below that reported for the methanol extract of the fruits of $F$. vasta for FL-cells (Al-Fatimi et al., 2007), which is a tumoral cell line derived from human amniotic epithelial cells, presenting a $\mathrm{CC}_{50}=980 \mu \mathrm{g} / \mathrm{mL}$. This indicates higher toxicity of the methanol extract of the aerial parts of $F$. vasta for Vero cells by the MTT method in relation to the toxicity of the methanol extract of its fruits for FL-cells by the neutral red method. On the other hand, the $\mathrm{CC}_{50}$ of $389 \mu \mathrm{g} / \mathrm{mL}$ in Vero cells observed in this study is higher than those reported for other extracts from plants with anticancer potential, such as Rheum ribes, Ficus bengalensis, Morus alba, Musa sapientum, Arnebia decumbens, Citrus limon, Fraxinus excelsior, Rumex acetosella and Arnebia echioides against 7 tumoral cell lines (Sardari et al., 2009), some of them from the Moraceae family. It is already reported that the cytotoxicity potential of compounds varies with the type of cell line and the method employed to evaluate it (Nogueira et al. 2011), also with the type of substance which is investigated and its time of exposition (Van de Sandt, 1992). The screening of anti-HSV-1 activity of the extract was carried on at the highest non-toxic concentration $(119 \mu \mathrm{g} / \mathrm{mL})$ in Vero cells, because being the viruses intracellular parasites, the death of the cells could lead to a false antiviral effect. The extract did not inhibit the development of the HSV-1 induced cytopathic effect (rounded cells and production of syncytium) at this concentration, as observed in figure 2. Regarding the compounds identified in the phytochemical characterization, there are reports of antiviral activity against HSV-1 for purified flavonoids quercetin and naningerin (Cotin et al., 2012) evaluated by the ortho-nitrophenyl- $\beta$-Dgalactopyranoside (ONPG) method, which measures the percentage of surviving cells in relation to an infected control. Differences in the methods employed to evaluate the antiviral activity as well as possible lower concentrations of these compounds in the methanol extract of $F$. vasta or even interactions of the different molecules present in the extract with them could perhaps explain the absence of anti-HSV-1 activity observed in this study. The screening of the antibacterial activity of the methanol extract of $F$. vasta aerial parts showed no inhibition of the growth of Gram-positive (S. aureus, S. epidermidis) or Gram-negative (E. coli, P. aeruginosa) bacteria, unlike the controls 20\% chlorhexidine and $0.05 \%$ enrofloxacine (figure 3). Concentrations above $119 \mu \mathrm{g} / \mathrm{mL}$ (highest nontoxic concentration in Vero cells) could be tested further aiming non-parenteral applications of the extract as antiseptic or disinfectant. This is supported by a report of antibacterial activity mainly against Gram-positive bacteria for an methanol extract from the fruits of $F$. vasta (Al-Fatimi et al., 2007), which was evaluated by the diskdiffusion method employing disks impregnated with 2 $\mathrm{mg}$ of the extract, despite the $1.19 \mu \mathrm{g}$ employed in this study.

\section{Phytochemical analysis}

Phytochemical analysis of methanol extract of F. vasta aerial parts revealed that it contained carbohydrates, tannins, flavonoids, coumarins, alkaloids and triterpenes (table 3). Chromatographic separation and purification of methanol extract of $F$. vasta allowed the identification of $\beta$ sitosterol, stigmasterol, lupeol, ursolic acid, naringenin, luteolin, quercetin, vitexin, quercetin $3-O-\beta$-glucoside and rutin (figure 4). Their structures were elucidated on the basis of UV, ${ }^{1} \mathrm{H}-\mathrm{NMR},{ }^{13} \mathrm{C}-\mathrm{NMR}$ and MS analyses.

\section{Structure elucidation of the isolated compounds} Data is given in table 2 .

\section{Identification of the active compounds of $F$. vasta methanol extract}

Chromatographic separation and purification of the methanol $80 \%$ extract of $F$. vasta methanol extract resulted in the isolation and identification of compound $1(\beta$ sitosterol) and compound 2 (stigmasterol) which gave dark spot under short UV light that changed to violet colour on spraying with vanillin sulphuric and heating in an oven at $110^{\circ} \mathrm{C}$ for $5 \mathrm{~min}$. NMR spectral data has shown signals very close to compound 1 ( $\beta$-sitosterol) and compound 2 (stigmasterol) (Pateh et al. 2009). Compound 3 (lupeol) gave a dark spot under short UV light and changed to pink to violet upon spraying with vanillinsulphuric acid and heating in an oven at $110^{\circ} \mathrm{C}$ for $5 \mathrm{~min}$. NMR spectral data showed signals very similar to lupeol (Abdullahi et al., 2013). Compound 4 (ursolic acid), was detected through spraying with vanillin sulphuric and heating in an oven at $110^{\circ} \mathrm{C}$ for $5 \mathrm{~min}$ and it gave violet spot at visible light, also NMR and MS spectral data are in accordance with published literature (Zuhal et al., 2006). Compound 5 (naringenin) was obtain as deep purple spot under UV light and changed to yellow when subjected to ammonia and $\mathrm{AlCl}_{3}$. Spectral data are very close to that described by Ibrahim et al. (2003). Compound 6 (luteolin) showed a deep purple spot under UV light which changed to yellow with ammonia vapor indicating that a flavone with free 5-OH and 4'-OH and spectral data of compound 6 is very close to that of (Owen et al. 2003). Compound 7 (quercetin) yellow spot and gave fluorescence yellow colour after spraying with $\mathrm{AlCl}_{3}$ and its spectral data are very similar to Manguro et al., 2005. Compound 8 (vitexin) gave deep purple spot under UV light and changed to yellow when subjected to ammonia and $\mathrm{AlCl}_{3}$. With complete acid hydrolysis, there is no change for compound 8 and thus, it was subjected to ferric chloride degradation, the products being chromatographed with authentic flavonoid aglycone and sugar samples, where apigenin as an aglycone and glucose moiety were detected and all spectral data were very close to that of (Yun-Lina et al., 2000). Compound 9 (quercetin 3 -O- $\beta$-glucoside) is obtained as deep purple spot and the compound gave yellow colour when exposed to ammonia vapour and gave a bright yellow colour when spraying with $\mathrm{AlCl}_{3}$. Complete acid hydrolysis of the compound gave quercetin as an aglycone and glucose as sugar moiety. Spectral data of this compound is very close to spectra of Song et al. (2007). Compound 10 (Rutin) gave a deep purple spot under UV light and changed to yellow when subjected to ammonia and $\mathrm{AlCl}_{3}$ and complete acid hydrolysis gave quercetin as an aglycone and glucose and 
rhamnose as sugar moieties and its spectral data was very similar to that of (Sintayehu et al., 2012).

\section{CONCLUSION}

This study evaluated the cytotoxicity of the methanol extract of the aerial parts of F. vasta in Vero cells, presenting a $\mathrm{CC}_{50}$ value which is intermediate among those already reported for $F$. vasta methanol extract of the fruits or extracts of some Moraceae species in different types of cell lines. This cytotoxicity determination will be important to be analyzed in the future if biological activities were found for this extract, because effective doses smaller than cytotoxic doses are desirable. There were not observed anti-HSV-1 activity and antibacterial activity against S. aureus, S. epidermidis, E. coli and P. aeruginosa at the highest non-toxic concentration of the extract in Vero cells by the methods employed in this study. Also this study gave a light on the main constituents of the extract, carbohydrates, tannins, flavonoids, coumarins, alkaloids and triterpenes and also identifies the bioactive compounds, $\beta$-sitosterol, stigmasterol, lupeol, ursolic acid, naringenin, luteolin, quercetin, vitexin, quercetin 3-O- $\beta$ glucoside and rutin from $F$. vasta methanol extract.

\section{CONFLICT OF INTEREST}

There is no conflict of interest associated with the authors of this paper.

\section{REFERENCES}

Abdullahi, S.M., Musa, A.M., Abdullahi, M.I., Sule, M.I., Sani, Y.M. (2013) Isolation of Lupeol from the Stem-bark of Lonchocarpus sericeus (Papilionaceae). Scholars Academic Journal of Biosciences 1(1):18-19. [DOI]

Abebe D, Debella A, Urga K (2003). Medicinal Plants and Other Useful Plants of Ethiopia. Ethiopian Health and Nutrition Research Institute, pp. 269-281. [DOI]

Al-Fatimi, M., Wurster, M., Schröder, G., Lindequist, U. (2007). Antioxidant, antimicrobial and cytotoxic activities of selected medicinal plants from Yemen. Journal of Ethnopharmacology 111: 657-666. [DOI]

Ali, N.A., Jülich, W.D., Kusnick, C., Lindequist, U. (2001). Screening of Yemeni medicinal plants for antibacterial and cytotoxic activities. Journal of Ethnopharmacology 74: 173-179. [DOI]

Bacon, T.H., Levin, M.J., Leary, J.J., Sarisky, R.T., Sutton, D. (2003). Herpes simplex virus resistance to acyclovir and pencyclovir after two decades of antiviral therapy. Clinical Microbiology Reviews 16: 114-28. [DOI

Cotin, S., Calliste, C.A., Mazeron, M.C., Hantz, S., Duroux, J.C., Rawlinson, W.D., Ploy, M.C., Alain, S. (2012). Eight flavonoids and their potential as inhibitors of human cytomegalovirus replication. Antiviral Research 96: 181-186. [DOI]

Denizot, F., Lang, R. (1986). Rapid colorimetric assay for cell growth and survival - modifications to the tetrazolium dye procedure giving improved sensitivity and reliability. Journal of Immunology Methods 89: 271-277. [DOI]

Hundera, K. (2007). Traditional Forest Management Practices in Jimma Zone, South West Ethiopia. Ethiopia Journal of Education Sciences 2(2): 1-5. [DOI]

Ibrahim, A.R.S., Galal, A.M., Ahmed, MS, Mossa, G.S. (2003). ODemethylation and Sulfation of 7-Methoxylated Flavanones by Cunninghamella elegans. Chemical Pharmaceutical Bulletin 51(2): 203-206. [DOI]

Lina, Y.L., Kuob, Y.H., Shiao, M.S., Chen, C.C., Oua, J.C. (2000). Flavonoid Glycosides from Terminalia catappa L. Journal of Chinese Chemical Society 47, 253-256. [DOI]

Manguro, L.O.A., Ugib, I., Lemmen, P. (2005). Flavonol Glycosides from the Leaves of Embelia keniensis. Journal of Chinese Chemical Society 52: 201-208. [DOI]

Morfin, F., Thouvenot, D. (2003). Herpes simplex virus resistance to antiviral drugs. Journal of Clinical Virology 26: 29-37. [DOI]

Nogueira DR, Mitjansa M, Infanteb MR, Vinardella MP (2011). Comparative sensitivity of tumor and non-tumor cell lines as a reliable approach for in vitro cytotoxicity screening of lysine-based surfactants with potential pharmaceutical applications. I. J. Pharm. 420: 51- 58. [DOI]
Owen, R.W., Haubner, R., Mier, W., Giacosa, A., Hull, W.E., Spiegelhalder, B., Bartsch, H. (2003). Isolation, structure elucidation and antioxidant potential of the major phenolic and flavonoid compounds in brined olive drupes. Food and Chemical Toxicology 41: 703-717. [DOI]

Pateh, U.U., Haruna, A.K., Garba, M., Iliya, I.M., Abubakar, M.S., Ambi, A.A (2009). Isolation of Stigmasterol, $\beta$-Sitosterol and 2-

Hydroxyhexadecanoic acid methyl ester from the Rhizomes of Stylochiton lancifolius and Kotchy (Araceae). Nigerian Journal of Pharmaceutical Sciences 7 (1): 19-25. [DOI]

Raju, N.J., Yesuf, E.A., Bekele, M., Wabe, N.T. (2011). Investigation of In Vitro Anthelmintic Activity of Ficus vasta leaves. Asian Journal of Pharmaceutical Biology 1(4): 454-458. [DOI]

Reed, L.J., Muench, H. (1938). A simple method of estimating fifty percent endpoints. American Journal of Hygine 27: 493-497. [DOI]

Sardari, S., Shokrgozar, M.A., Ghavami, G. (2009). Cheminformatics based selection and cytotoxic effects of herbal extracts. Toxicology in vitro 23 : 1412-1421. [DOI]

Sintayehu, B., Asres, K., Raghavendra, Y. (2012). Radical scavenging activities of the leaf extracts and a flavonoid glycoside isolated from Cineraria abyssinica. Sch. Bip. Exa. Rich. Journal of Applied Pharmaceutical Sciences 2 (4): 44-49. [DOI]

Song, N., Xu, W, Guan,. H., Liu, X., Wang, Y., Nie, X. (2007). Several flavonoids from Capsella bursa-pastoris (L.) Medic. Asian Journal of Traditional Medicines 2(5): 218-22. [DOI]

Van de Sandt, J.J.M., Rutten, A.A.J.J.L., Koëter, H.B.W.M. (1993). Cutaneous toxicity testing in organ culture: neutral red uptake and reduction of tetrazolium salt (MTT). Toxicology in vitro 7 (1): 81-86. [DOI]

Yadav, R.N.S., Agarwala, M. (2011). Phytochemical analysis of some medicinal plants. Journal of Phytology 3(12): 10-14. [DOI]

Zuhal, G., Nurcan, K., Cavit, K., Yusuf, K., Omur, L.D. (2006). Chemical Constituents of Galium tortumense. Turk J Chem 30:515-523. [DOI] 